\title{
Usability assessment of a Bluetooth-enabled resistance exercise band among young adults
}

\author{
Lillian M. Seo ${ }^{1}$, Curtis L. Petersen ${ }^{2,3}$, Ryan J. Halter ${ }^{4}$, David F. Kotz ${ }^{5}$, Karen L. Fortuna ${ }^{1,6}$, \\ John A. Batsis ${ }^{1,2,7,8} \wedge$
}

${ }^{1}$ Geisel School of Medicine at Dartmouth, Hanover, New Hampshire, USA; ${ }^{2}$ The Dartmouth Institute for Health Policy \& Clinical Practice, Lebanon, New Hampshire, USA; ${ }^{3}$ Department of Quantitative Biomedical Science, Dartmouth College, USA; ${ }^{4}$ Thayer School of Engineering, Dartmouth College, USA; ${ }^{5}$ Department of Computer Science, Dartmouth College, USA; ${ }^{6}$ Department of Psychiatry, Dartmouth Hitchcock, Lebanon, New Hampshire, USA; ${ }^{7}$ Department of Medicine, Dartmouth Hitchcock, Lebanon, New Hampshire, USA; ${ }^{8}$ Division of Geriatric Medicine and Department of Nutrition, Gillings School of Global Public Health, University of North Carolina at Chapel Hill, Chapel Hill, North Carolina, USA

Contributions: (I) Conception and design: LM Seo, CL Petersen, JA Batsis; (II) Administrative support: JA Batsis, RJ Halter, DF Kotz; (III) Provision of study materials or patients: LM Seo, JA Batsis; (IV) Collection and assembly of data: LM Seo, CL Petersen, RJ Halter, DF Kotz; (V) Data analysis and interpretation: LM Seo, CL Petersen, JA Batsis; (VI) Manuscript writing: All authors; (VII) Final approval of manuscript: All authors.

Correspondence to: John A. Batsis, MD. Associate Professor, 5017 Old Clinic Building, Division of Geriatric Medicine, University of North Carolina at Chapel Hill, Chapel Hill, NC, USA. Email: John.batsis@gmail.com.

Background: Resistance-based exercises effectively enhance muscle strength, which is especially important in older populations as it reduces the risk of disability. Our group developed a Bluetooth-enabled handle for resistance exercise bands that wirelessly transmits relative force data through low-energy Bluetooth to a local smartphone or similar device. We present a usability assessment that evaluates an exercise system featuring a novel Bluetooth-enabled resistance exercise band, ultimately intended to expand the accessibility of resistance training through technology-enhanced home-based exercise programs for older adults. Although our target population is older adults, we assess the user experience among younger adults as a convenient and meaningful starting point in the testing and development of our device.

Methods: There were 32 young adults participating in three exercise sessions with the exercise band, after which each completed an adapted version of the Usefulness, Satisfaction, and Ease (USE) questionnaire to characterize the exercise system's strengths and weaknesses in usability.

Results: Questionnaire data reflected a positive and consistent user experience, with all 20 items receiving mean scores greater than 5.0 on a seven-point Likert scale. There were no specific areas of significant weakness in the device's user experience.

Conclusions: The positive reception among young adults is a promising indication that the device can be successfully incorporated into exercise interventions and that the system can be further developed and tested for the target population of older adults.

Keywords: Exercise; mHealth; resistance training; telerehabilitation

Received: 12 June 2020; Accepted: 11 January 2021; Published: 30 April 2021.

doi: $10.21037 /$ ht-20-22

View this article at: http://dx.doi.org/10.21037/ht-20-22

^ ORCID: 0000-0002-0845-4416. 


\section{Introduction}

Resistance-based exercises promote musculoskeletal health and functional performance, which become especially important for older individuals to address the natural processes of aging including loss of muscle and increased adipose tissue $(1,2)$. Challenges surrounding accessibility make it difficult for many older individuals to consistently adhere to traditional exercise programs $(3,4)$, which in turn correlates to reduced exercise effectiveness and functional outcomes (5-7). Various technologies enabling remote monitoring help address many of the barriers that affect exercise adherence while providing functional data, which can inform both users and their supervising clinicians when they engage in home-based exercise programs $(5,8)$. Younger adults are generally more technologically adept compared to older adults (9) and thus represent a convenient starting point in characterizing and improving upon the user experience of a new device. Positive reception among younger adults signals that the device may reasonably be developed for older populations.

Our group developed a remote sensing, Bluetooth-connected resistance exercise band that captures and transmits relative force data $(10,11)$. The device comprises a commercially available Theraband and a Tekscan FlexiForce A201 sensor attached to one of the device's semi-rigid handles (Figure 1). As the user pulls on the handles to perform an exercise, the sensor measures relative force exerted on the handles and transmits the data to a nearby smartphone via low-energy Bluetooth at a rate of 10 readings per second (10-12). This system has the potential to enhance home-based exercise programs by allowing health professionals to monitor engagement and adherence to home-based exercise regimens $(13,14)$. Theoretically, measuring performance quantitatively can help achieve consistent and highquality exercise completion, although not directly examined by this study. The device's basic functioning and usability has already been established in both clinical and field-based settings (12), though its usability with repeated use has not been fully explored. In this study, we assess the usability of this novel device by deploying it among younger adults with repeated usage over three exercise sessions. Our intent was to characterize the user experience of the system in advance of testing and improving its design for its intended application in older adults.

\section{Methods}

\section{Experimental approach}

We conducted a prospective cohort usability study with 32 participants using a system featuring a Bluetooth-enabled
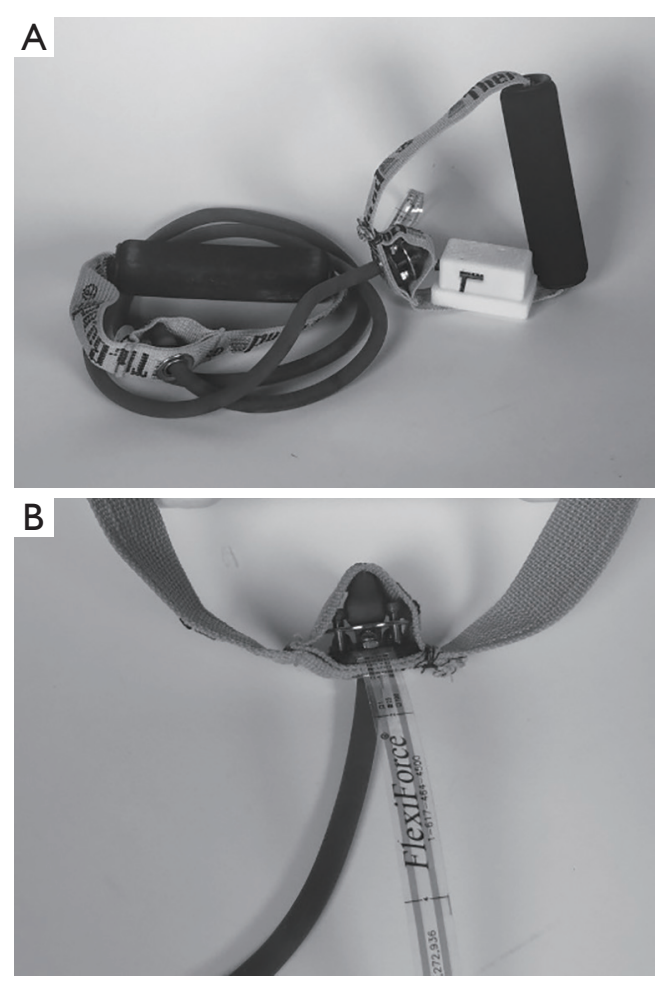

Figure 1 Bluetooth-enabled resistance exercise band that enables force monitoring and real-time data transmission to a local smart device. (A) Device featuring a commercially available Theraband with an attached force sensor and on/off switch. (B) Force sensor attached to one handle that measures relative force applied as the user pulls on the handles to perform exercises.

exercise band. All study participants repeatedly used the exercise band for three exercise sessions. Sessions lasted about 15-20 minutes each and were separated by at least 24 hours to minimize muscle fatigue. They were performed in community-based settings, most often in the participants' places of residence. Before the first session, participants completed a validated Basic Fitness Level questionnaire to characterize self-perceived current physical condition, composed of 12 items on a five-point Likert scale ( 1 = "strongly disagree", 5 = "strongly agree") (15). After the final exercise session, participants completed an adapted version of the Usefulness, Satisfaction, and Ease (USE) questionnaire (16), a validated tool that evaluates the system and user experience across four domains: usefulness, satisfaction, ease of use, and ease of learning. The questionnaire was adapted for brevity by excluding 10 items that were not applicable to our device. The adapted version consisted of 20 positive statements regarding the system's usability, to which participants indicated their 


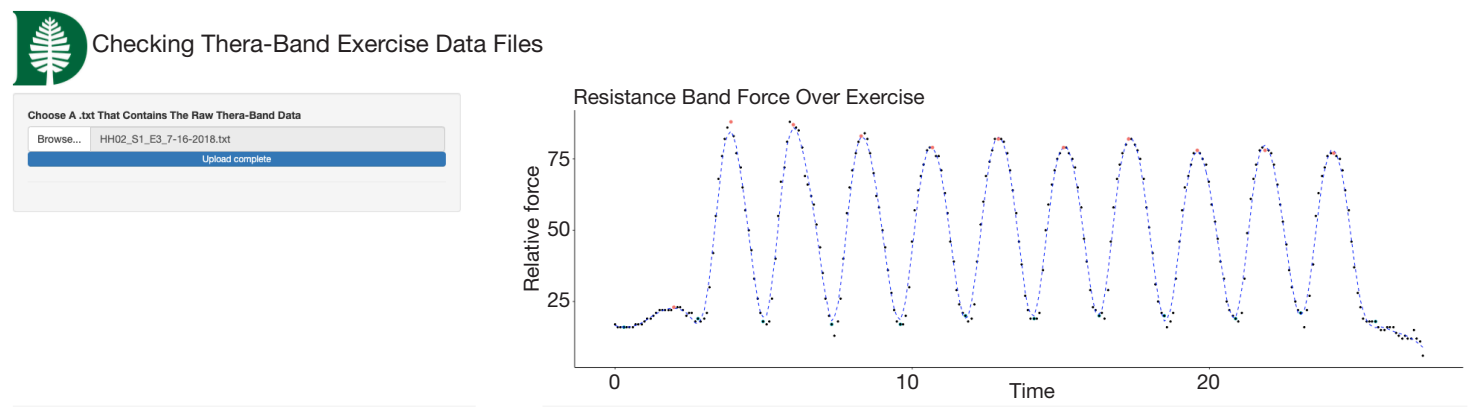

Author: Gurtis L. Petersen

Figure 2 Visualization of data with custom web app depicting number of exercise repetitions performed and relative force exerted, for a single exercise from one session by a participant. Each point is the relative force exerted at a given point in time with time points connected by a blue dotted line.

degree of agreement on a seven-point Likert scale $(1=$ "very strongly disagree", 7 = "very strongly agree"). We aggregated data by computing mean response values across participants for each question and domain. We evaluated for associations between questionnaire responses and age as well as gender by performing correlations between USE scores with age, and a two-tailed independent t-test between genders. An optional prompt at the end of the questionnaire solicited openended feedback to gain additional qualitative indicators of usability and generate ideas for design improvement. Finally, we processed the force data with a custom web application developed through R Shiny, allowing us to visualize the number of exercise repetitions as well as the relative force exerted on the handle during exercise completion. In brief, the application's algorithm uses a running window function over a local window regression of the collected force overtime and identifies peaks and troughs in the data curves (17) (Figure 2). The fidelity of the system's ability to detect number of repetitions was assessed qualitatively by ensuring that Bluetooth-captured data was observable through the web application. The application verified that useable data was being collected by displaying (I) force collected by the device, (II) a smoothed line of the force, and (III) peaks and troughs of the force with one exercise repetition represented by two successive peaks. We video-recorded the exercise sessions to help us later validate the accuracy of data processing by the web application.

\section{Subjects}

We recruited participants who were 18-65 years old and without physical limitations preventing them from performing any of the exercises. Of the 32 participants, 13 (41\%) were female; mean age was $32.4 \pm 11.8$ years. The Basic Fitness Level questionnaire demonstrated that no participants had a physical impairment and their self-perceived physical conditions were high, with the mean score of the item "I am in good physical condition" being $4.16 \pm 0.64$ out of 5 . The study was conducted in accordance with the Declaration of Helsinki (as revised in 2013) and approved by Dartmouth-Hitchcock Institutional Review Board \#31400. Written informed consent was taken from all the participants.

\section{Procedures}

Participants received a brief, interactive teaching session on how to use this device and perform four basic exercises: bicep flexion, shoulder abduction, seated row, and tricep extension (Figure 3). Participants learned proper technique, posture, and alignment and practiced using the handle's simple on/off switch. The first of the exercise sessions began after establishing comfort with the device and exercises. During each session, participants performed 10 repetitions of each exercise with a period of rest between each exercise set if needed. The handle transmitted measurements of the force exerted by the exercise band to a nearby smartphone in real-time.

\section{Results}

On the USE questionnaire, all 20 items received mean scores greater than 5.0 out of 7 , with 15 items having mean scores greater than 6.0 (Table 1). The two lowest-scoring items posed the statements "The Theraband helps me be more effective" 
A

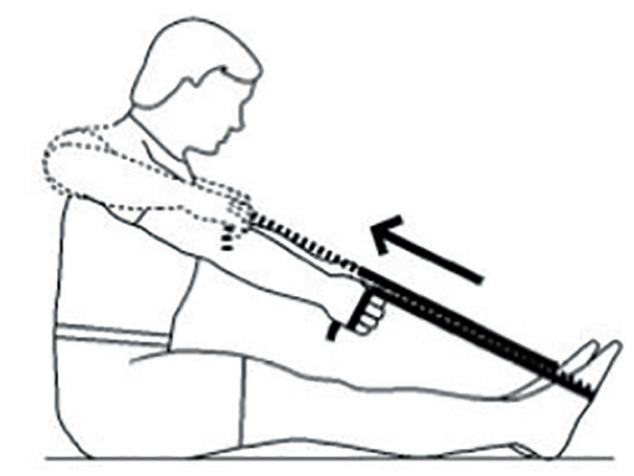

C

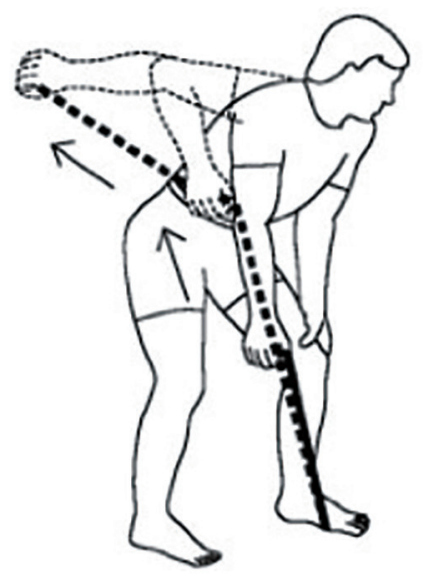

B
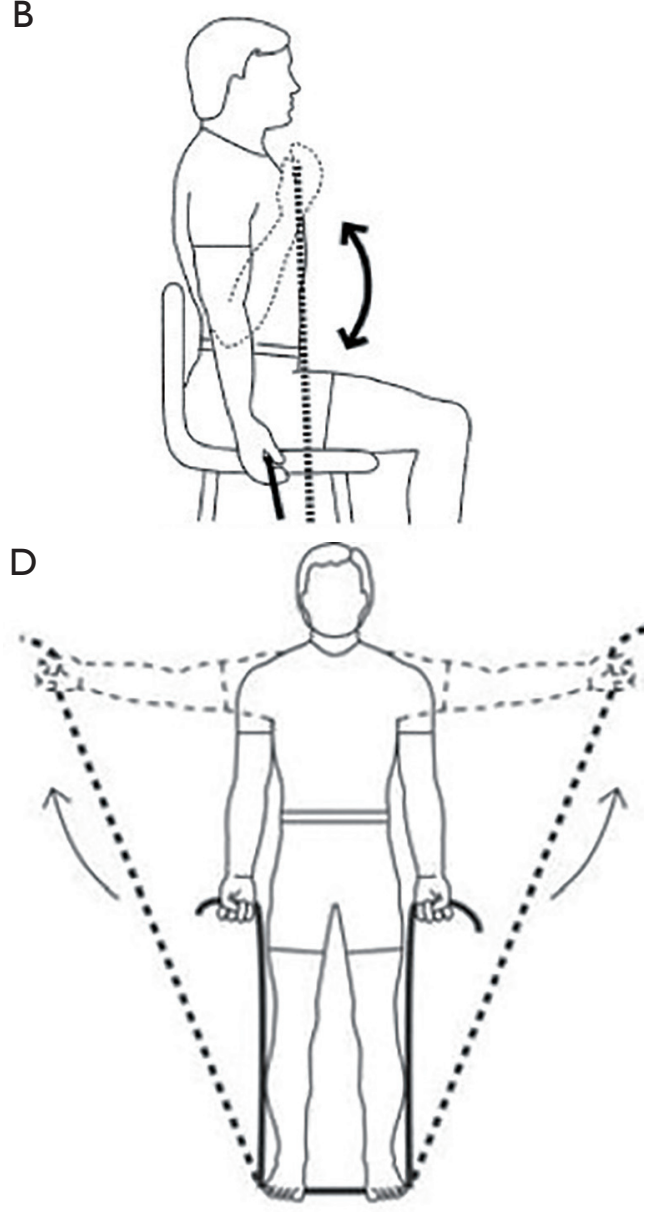

Figure 3 Four exercises performed with resistance exercise band (12): (A) seated row, (B) bicep flexion, (C) tricep extension, (D) shoulder abduction.

and "Using the Theraband is effortless" with mean scores of 5.25 and 5.19, respectively. The mean scores of the four usability domains were: usefulness $5.63 \pm 0.33$; satisfaction $6.19 \pm 0.03$; ease of use $6.21 \pm 0.45$; and ease of learning $6.48 \pm 0.16$. There was no significant correlation or association between USE scores and age $(r=-0.08)$ or gender $(\mathrm{P}=0.48)$, respectively. Openended feedback was limited as not all participants chose to leave comments, but general impressions were favourable and supported usability as a strength of the system. Representative quotes included: "A non-rigid strap-style bandle might be useful for some exercises," and "Would need different bands for each exercise as they use different muscle groups with different strength levels."

\section{Discussion}

The results from our usability assessment suggest that the user experience of our system is consistent and positive among younger adults. This outcome is encouraging and indicates value in further developing this device for deployment among older adults.

The system's strengths included its ease of use, ease of learning, and user satisfaction with each of these domains receiving mean scores greater than 6.0 out of seven. Relatively low scores on a few questionnaire items may in part be explained by the wording and interpretation of statements. For example, in the statement "Using the Theraband is effortless", it was possible that some participants interpreted the meaning of effort as reflecting physical exertion rather than ease of use from a usability standpoint. Thus, the relatively low mean score of this item might reflect a different metric than what was intended by the questionnaire. In future research regarding strength and 
Table 1 Mean response values on adapted USE questionnaire

\begin{tabular}{|c|c|c|}
\hline Domain & USE questionnaire statement & Mean response value \pm SD \\
\hline \multirow{2}{*}{ Usefulness } & The Theraband is useful & $5.75 \pm 1.16$ \\
\hline & The Theraband does everything I would expect it to do & $5.88 \pm 1.18$ \\
\hline \multirow[t]{7}{*}{ Ease of use } & The Theraband is easy to use & $6.47 \pm 0.76$ \\
\hline & The Theraband is user-friendly & $6.41 \pm 0.84$ \\
\hline & The Theraband is flexible & $6.75 \pm 0.51$ \\
\hline & Using the Theraband is effortless & $5.19 \pm 1.40$ \\
\hline & I can use the Theraband without written instructions & $5.78 \pm 1.56$ \\
\hline & I can recover from mistakes quickly and easily & $6.34 \pm 0.83$ \\
\hline & I can use the Theraband successfully every time & $6.25 \pm 0.80$ \\
\hline \multirow[t]{4}{*}{ Ease of learning } & I learned to use the Theraband quickly & $6.63 \pm 0.55$ \\
\hline & I easily remember how to use the Theraband & $6.38 \pm 0.98$ \\
\hline & It is easy to learn to use the Theraband & $6.63 \pm 0.55$ \\
\hline & I quickly became skillful with the Theraband & $6.31 \pm 0.89$ \\
\hline \multirow[t]{2}{*}{ Satisfaction } & I am satisfied with the Theraband & $6.16 \pm 0.95$ \\
\hline & I would recommend the Theraband to a friend & $6.22 \pm 1.0$ \\
\hline
\end{tabular}

1: very strongly disagree; 2: strongly disagree; 3: disagree; 4: neutral; 5: agree; 6: strongly agree; 7: very strongly agree.

conditioning, we recommend rewording such questions to avoid this confusion.

The data indicate a well-rounded user experience with the lowest scoring items and domains still with mean scores greater than 5.0. On a seven-point Likert scale where a value of five represents "Agree," these mean scores still represent agreement with the questionnaire items and reflect positive perspectives on the system's usability. The open-ended comments, though few in number, generated ideas for improvements to the device's physical design to make it more suitable for home-based exercise interventions. Finally, data visualization with a web application demonstrated that the force sensor attached to the band enables monitoring of two key aspects of exercise sessions: number of repetitions performed, and relative force exerted on the band. Visualization of force data demonstrated fidelity of the system's data capturing capability and supported that quantitative monitoring of exercise sessions is feasible with our system.

Though promising, our study is not without limitations. A formal quantitative assessment of the system's accuracy in detecting the number of exercise repetitions can be considered in future studies with larger test datasets. In addition, it is unclear whether these results will generalize to the independent, unsupervised setting of home-based exercise programs. Our sample was relatively homogenous in that all participants had high baseline fitness levels and no physical impairments. Lower fitness levels and physical limitations may significantly impact the user experience for other individuals. Lastly, we have yet to deploy the device among older adults with repeated usage. There may be barriers or considerations unique to older adults that will only be revealed with a usability assessment dedicated to this target population. 


\section{Conclusions}

In summary, our device featuring a novel Bluetoothconnected resistance exercise band represents a potential exercise system particularly suited for older individuals and home-based exercise programs. This study provides adequate and encouraging formative data on the system's usability among young adults, assuring that next steps can be taken to continue testing and development for the target population of older adults. Ultimately, our system will provide a safe and effective exercise mode with usability as a major strength as well as reliable performative feedback, bringing the health benefits of strength-based exercise to individuals of all ages.

\section{Acknowledgments}

Funding: Mr. Petersen is supported by the BurroughsWellcome Fund: Big Data in the Life Sciences at Dartmouth. This work was funded in part by The Center for Technology and Behavioral Health at Dartmouth pilot grant under NIDA Grant Number P30DA0229926. Dr. Batsis' research reported in this publication was supported in part by the National Institute on Aging of the National Institutes of Health under Award Number K23AG051681. Professors Halter and Kotz were supported by the National Science Foundation under award numbers CNS-1314281 and CNS-1619970. Drs. Batsis and Halter have submitted a preliminary patent application 62/672,827 titled Instrumented Resistance Exercise Device and patent titled Remote Sensing, Bluetooth-enabled resistance exercise band: Patent Application No. 63033022, filed June 1st, 2020. Dr. Fortuna was funded through a National Institute of Mental Health K01 award (K01MH117496). The content is solely the responsibility of the authors and does not necessarily represent the official views of the sponsors.

\section{Footnote}

Data Sharing Statement: Available at http://dx.doi. org/10.21037/ht-20-22

Peer Review File: Available at http://dx.doi.org/10.21037/ht20-22

Conflicts of Interest: All authors have completed the ICMJE uniform disclosure form (available at http://dx.doi. org/10.21037/ht-20-22). Dr. LMS reports having a patent instrumented resistance band issued, and a patent Remote sensing Bluetooth exercise band pending. Mr. CLP reports having a patent 63/033,022 pending to Dartmouth College and an equity in a group that develops resistant band technology. Dr. RJH reports grants from National Science Foundation, grants from National Science Foundation, during the conduct of the study; In addition, Dr. RJH has a patent Instrumented Resistance Exercise Device: Patent Application No. 62/672,827 pending. Dr. KLF reports grants from National Institute of Mental Health, during the conduct of the study. Dr. JAB reports grants from NIH, during the conduct of the study; personal fees from Dinse, Knapp McAndrew, personal fees from NIH, outside the submitted work. In addition, Dr. JAB has a patent Instrumented Resistance Exercise Device (Patent Application No. 62/672,827) and a pending patent 63/033,022. Mr. CLP, Dr. RJH, and Dr. JAB all have equity in a SynchroHealth LLC, a group that develops resistance band technology.

Ethical Statement: The authors are accountable for all aspects of the work in ensuring that questions related to the accuracy or integrity of any part of the work are appropriately investigated and resolved. The study was conducted in accordance with the Declaration of Helsinki (as revised in 2013) and approved by Dartmouth-Hitchcock Institutional Review Board \#31400. Written informed consent was taken from all the participants.

Open Access Statement: This is an Open Access article distributed in accordance with the Creative Commons Attribution-NonCommercial-NoDerivs 4.0 International License (CC BY-NC-ND 4.0), which permits the noncommercial replication and distribution of the article with the strict proviso that no changes or edits are made and the original work is properly cited (including links to both the formal publication through the relevant DOI and the license). See: https://creativecommons.org/licenses/by-nc-nd/4.0/.

\section{References}

1. Lopez P, Pinto RS, Radaelli R, et al. Benefits of resistance training in physically frail elderly: a systematic review. Aging Clin Exp Res 2018;30:889-99.

2. Schoenfeld BJ, Ogborn D, Krieger JW. Effects of Resistance Training Frequency on Measures of Muscle Hypertrophy: A Systematic Review and Meta-Analysis. Sports Med 2016;46:1689-97. 
3. Campbell R, Evans M, Tucker M, et al. Why don't patients do their exercises? Understanding non-compliance with physiotherapy in patients with osteoarthritis of the knee. J Epidemiol Community Health 2001;55:132-8.

4. Freiberger E, Kemmler W, Siegrist M, et al. Frailty and exercise interventions: Evidence and barriers for exercise programs. Z Gerontol Geriatr 2016;49:606-11.

5. Argent R, Daly A, Caulfield B. Patient Involvement With Home-Based Exercise Programs: Can Connected Health Interventions Influence Adherence?. JMIR Mhealth Uhealth 2018;6:e47.

6. O'carroll M, Hendriks O. Factors associated with rheumatoid arthritis patients' compliance with home exercises and splint use. Physiother Theory Pract 1989;5:115-22.

7. Sluijs EM, Kok GJ, van der Zee J. Correlates of exercise compliance in physical therapy. Phys Ther 1993;73:771-82; discussion 783-6.

8. Caulfield BM, Donnelly SC. What is connected health and why will it change your practice? QJM 2013;106:703-7.

9. Czaja SJ, Charness N, Fisk AD, et al. Factors predicting the use of technology: Findings from the Center for Research and Education on Aging and Technology Enhancement (CREATE). Psychol Aging 2006;21:333-52.

10. Mohieldin S. BANDPASS: A Smart Resistance Exercise Band to Monitor Strength. Dartmouth Technical Report. Available online: https://digitalcommons.dartmouth.edu/

doi: $10.21037 /$ ht-20-22

Cite this article as: Seo LM, Petersen CL, Halter RJ, Kotz DF, Fortuna KL, Batsis JA. Usability assessment of a Bluetoothenabled resistance exercise band among young adults. Health Technol 2021;5:4. cgi/viewcontent.cgi? article $=1010 \&$ context=engs 88

11. Wechsler EV. Development of a "Smart" Resistance Exercise Band to Assess Strength. Senior Honors Thesis. Available online: https://digitalcommons.dartmouth.edu/ wetterhahnsymposium-2018/5/

12. Batsis JA, Boateng GG, Seo LM, et al. Development and Usability Assessment of a Connected Resistance Exercise Band Application for Strength-Monitoring. World Acad Sci Eng Technol 2019;13:340-8.

13. Palazzo C, Klinger E, Dorner V, et al. Barriers to homebased exercise program adherence with chronic low back pain: Patient expectations regarding new technologies. Ann Phys Rehabil Med 2016;59:107-13.

14. Pisters MF, Veenhof C, Schellevis FG, et al. Exercise adherence improving long-term patient outcome in patients with osteoarthritis of the hip and/or knee. Arthritis Care Res (Hoboken) 2010;62:1087-94.

15. Abadie BR. Construction and Validation of a Perceived Physical Fitness Scale. Percept Mot Skills 1988;67:887-92.

16. Lund AM. Measuring Usability with the USE Questionnaire. Available online: http://edutechwiki.unige. ch/en/Usability_and_user_experience_surveys

17. Petersen CL, Wechsler EV, Halter RJ, et al. Detection and Monitoring of Repetitions Using an mHealth-Enabled Resistance Band. IEEE Int Conf Connect Health Appl Syst Eng Technol 2018;2018:22-4. 